\section{Triplication of a short arm region of chromosome 15}

SIR,

A recent case report by Watson and Scrimgeour (1977) described an abnormal chromosome 15 in amniotic fluid cells, which appeared to consist of a dicentric element with 2 satellite regions. This abnormal chromosome appeared to have no clinical significance since it was present in each of 2 apparently normal male twin fetuses and also in the carrier mother. The chromosome was stable and G- and C-banding studies suggested that it was a dicentric element with the lower centromere inactive and prematurely divided. The development of silver staining techniques to highlight nucleolar organising (NO) regions (Bloom and Goodpasture, 19.76), and the recent introduction of the Distamycin A + DAPI technique (Schweizer et al., 1978), which specifically stains band p11 of chromosome 15 (Spowart, 1978), prompted us to re-examine slides prepared from blood samples of the carrier mother. Slides were stained using G-, Q-, C-, and Rbanding as well as DA-DAPI and silver NO techniques.

C-band preparations showed 3 darkly stained regions in the short arm-centromeric region of the abnormal chromosome 15,2 of which were originally considered to be regions of centromeric heterochromatin (Fig. 1b. of Watson and Scrimgeour, 1977) associated with 2 regions of satellite material, whose presence was deduced from satellite association patterns. Our studies with silver staining showed tha there is no evidence of an active NO region on the short arm of the chromosome, though, as shown by Watson and Scrimgeour, it does form associations with other acrocentric chromosomes and presumably consists of normal short arm material with an inactive NO region. There is, however, a large active NQ: region distal to the centromere, approximately one third of the distance along the length of the long arri (Fig.).

The DA-DAPI technique produces bright fluorescence at the $\mathrm{C}$-band regions of chromosomes $1+$ 9 , and 16, at the long arm of the Y chromosome, and at band p11 of chromosome 15 (Schweizer et al. $\overrightarrow{\text { H }}$ 1978), whereas the stalk region (p12) or the satellit? (p13) of chromosome 15 are not fluorescent (Spowarto 1978). With this technique, the abnormal 15 is found to possess 3 bright bands (Fig.) which, taken in conjunction with the other banding results, indicates the presence of three bands p11. The patient's karyotype could therefore be interpreted as $46, \mathrm{XX}$,trio (15) (pter or p12 $\rightarrow$ cen $\rightarrow$ p12::p12 $\rightarrow$ qter). A less likels alternative of tandem repeats would be $46, X X, \operatorname{tri}(15$ (pter or p12 $\rightarrow$ cen::p12 or p11 $\rightarrow$ cen::p12 $\rightarrow$ qter). WE cannot state conclusively whether there are 1,2 , or $\$$ centromere regions on this chromosome, but there is clearly only 1 functional centromere.

The presence of this triplicated region oD chromosome 15 is interesting as we have noted that is several of the cases presenting with a small accessory

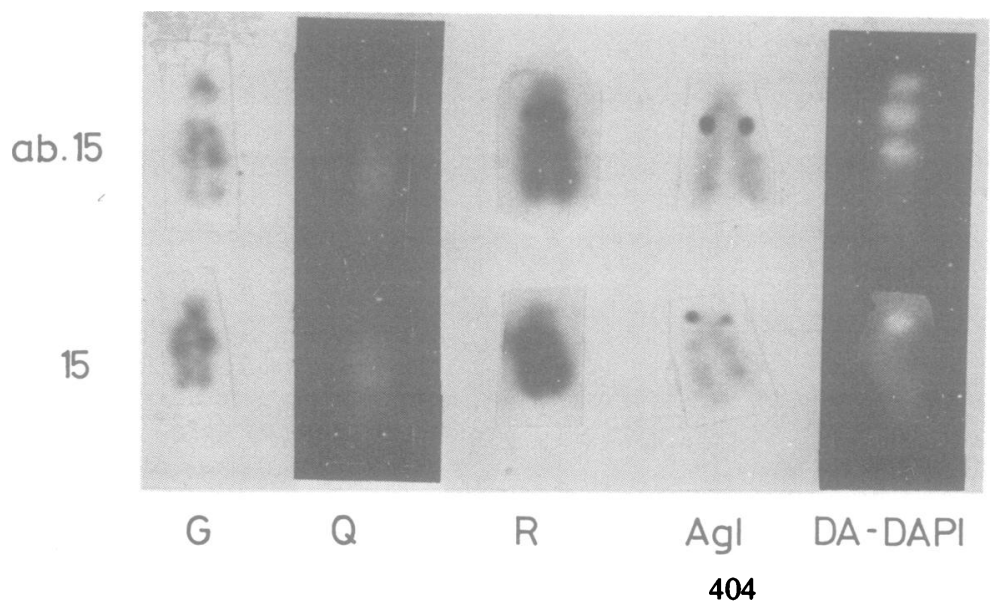

$G$-, $Q$-, and $R$-banding, NO silve $\bar{\complement}$ staining, and Distamycin $A$ plus. DAPI staining of abnormal and normal no. 15 chromosomes. 
or supernumerary element, when examined with the silver NO and DA-DAPI techniques, the supernumerary is shown to consist of $15 \mathrm{p}$ plus centromere joined to another 15p. Such chromosomes have been observed in individuals with normal, as well as abnormal, phenotypes.

I am grateful to Dr J. Scrimgeour for providing a blood sample for cytogenetic studies and to Miss $\mathrm{K}$. Buckton and Professor H. J. Evans for helpful discussion.

\section{G. SPOWART MRC Clinical and Population Cytogenetics Unit, Western General Hospital, Edinburgh}

\section{References}

Bloom, S. E., and Goodpasture, C. (1976). An improved technique for selective silver staining of nucleolar organizer regions in human chromosomes. Human Genetics, 34, 199-206.

Schweizer, D., Ambros, P., and Andrle, M. (1978). Modifcation of DAPI banding on human chromosomes by prestaining with a DNA-binding oligopeptide antibiotic, Distamycin A. Experimental Cell Research, 111, 327-332.

Spowart, G. (1978). Reassessment of presumed $Y / 22$ and $Y / 15$ translocations in man using a new technique. Cytogenetics and Cell Genetics. (In the press.)

Watson, E. J., and Scrimgeour, J. B. (1977). A dicentric no. 15 chromosome with two satellite regions. Journal of Medical Genetics, 14, 381-383.

\section{Placentation, anencephaly, and spina bifida}

SIR,

I want to suggest that the immediate cause of anencephaly and spina bifida (ASB) is typically a dietary or hormonal deficiency in the embryo brought about by defective placentation. (It should be noted that the word 'placenta' here denotes the system of nutrition immediately after implantation. Logically, the hypothesis does not require the existence of an organ of that name.)

Concordance rates for anencephaly in twins are modest. In a review of affected pairs ascertained in series, 13 of 309 same-sexed pairs, and 3 of 118 opposite-sexed pairs, were reportedly concordant (James, 1978). These concordance rates were estimated to be slightly higher than would be expected on the basis of (i) the raised recurrence rates in the sibs of affected cases; and (ii) the hypothesised higher rates in monozygotic twins (James, 1976). Nevertheless, the cause would seem to be shared only to a small extent by both members of a twin pair. At first sight this seems a very odd fact as it apparently rules out genetic causes. Moreover, it is not easy to see how the
2 members of a twin pair can be unequally exposed to an environmental teratogen. The explanation, I suggest, lies in the placentation: twin members do not always share the same placenta and, even when they do, their facilities are often unequal.

If one accepts that the teratogen is dietary or hormonal in nature (and most of the points to be presented seem good evidence for such a view), then the only logical alternative to this placenta hypothesis seems to be that the teratogen is very powerful, but exists in such tiny quantities (a few molecules) in the maternal circulation that only one twin is usually affected. I can think of no good ground for this alternative, whereas the following points seem to implicate the placenta.

(1) Anencephaly is closely associated with defective placentation. Berge (1965) examined the placentae of 10 anencephalic fetuses and found that all of them contained areas of degeneration and scar tissue. Benirschke and Driscoll (1967) offer evidence that placental infarcts are of maternal, rather than fetal, origin, and hence are not to be interpreted as an effect of the malformed fetus, but possibly as a cause.

(2) Placenta praevia has been reported to be common in anencephalic maternities (Smilkstein, 1962; Smithells et al., 1964). Record and McKeown (1949) estimated that the incidence of placenta praevia in anencephalic maternities is 10 times that in control maternities. Again, it seems reasonable to suppose that this condition predates (and therefore may somehow cause) the malformation.

(3) It has also been reported that anencephaly is associated with a single umbilical artery (Cipparone, 1966). This is another condition in which the fetus is suspected of being deprived of nutrient (Benirschke and Driscoll, 1967).

(4) There is the observation (Talbot, 1924) that in a pair of twins discordant for anencephaly, the affected twin had a placenta that was infarcted, whereas the other placenta was normal.

(5) It has been noted (Mall, 1908) that ASB is disproportionately common in extrauterine pregnancy, a condition in which the placenta is usually defective.

(6) This placenta hypothesis would explain the apparently higher concordance rates in samesexed than in opposite-sexed affected pairs. This phenomenon would be partially (but not wholly) explained by the hypothesised higher incidence rates in monozygotic twins (James, 1976). The additional explanation now being offered is that monozygotic pairs sometimes share the same defective placenta.

There is plenty of evidence (Coffey and Jessop, 